\title{
Up-regulation of neutrophil gelatinase-associated lipocalin in oral squamous cell carcinoma: Relation to cell differentiation
}

\author{
TAKASHI HIROMOTO, KAZUMA NOGUCHI, MICHIYO YAMAMURA, YUSUKE ZUSHI, EMI SEGAWA, \\ KAZUKI TAKAOKA, KUNIYASU MORIDERA, HIROMITSU KISHIMOTO and MASAHIRO URADE
}

\author{
Department of Oral and Maxillofacial Surgery, Hyogo College of Medicine, \\ 1-1 Mukogawa-cho, Nishinomiya, Hyogo 663-8501, Japan
}

Received January 19, 2011; Accepted February 23, 2011

DOI: 10.3892/or.2011.1429

\begin{abstract}
Neutrophil gelatinase-associated lipocalin (NGAL, also known as lipocalin2, LCN2) is a secreted glycoprotein with increased expression in solid tumors. The expression and functions of NGAL in oral cancer, however, remain unclear. We investigated the expression of NGAL in oral cancer tissues and oral cancer cell lines. By immunohistochemical examinations, NGAL expression was strongly up-regulated in well-differentiated OSCC tissues and moderately to weakly up-regulated in moderately to poorly differentiated OSCC tissues. In contrast, NGAL expression was weak or very weak in normal mucosa and leukoplakia. By Western blot analysis, NGAL expression levels positively correlated with cell morphology patterns and loss of E-cadherin. In addition, the enzymatic activity of the NGAL/MMP-9 complex significantly correlated with the results obtained by zymographic analysis. In conclusion, NGAL expression is high in welldifferentiated cancer, suggesting that NGAL may be a useful diagnostic marker of tumor-cell differentiation.
\end{abstract}

\section{Introduction}

Cancer is a multistep process during which cells undergo alterations of their normal function, progressively leading to tumorigenesis (1). Among the most important changes, cancer cells begin to secrete a number of factors into the tumor microenvironment. These factors stimulate tumor growth and survival and mediate angiogenesis and metastasis. Oral squamous cell carcinoma (OSCC) is one of the most common neoplasms in the world, characterized by a poor prognosis and low survival rate $(2,3)$. The GLOBOCAN 2002 database compiled by the International Agency for Cancer Research (IARC) estimated that the worldwide annual numbers of

Correspondence to: Dr Kazuma Noguchi, Department of Oral and Maxillofacial Surgery, Hyogo College of Medicine, 1-1 Mukogawa-cho, Nishinomiya, Hyogo 663-8501, Japan

E-mail: knoguchi@hyo-med.ac.jp

Key words: oral cancer, neutrophil gelatinase-associated lipocalin, lipocalin2, differentiation newly diagnosed cases of oral cancer and of oral-cancerrelated deaths amount to 274,000 and 127,000 , respectively (4). OSCC results from the cumulative effects of genetic alterations, transforming normal cells into precancerous cells and then cancer cells through a series of progressive steps. However, the detailed mechanisms of oral carcinogenesis remain unclear. Nevertheless, an understanding of the pathogenesis of OSCC, accompanied by the development of new therapeutic methods and diagnostic techniques that promote early detection, may facilitate effective prevention and treatment, thereby improving survival.

Neutrophil gelatinase-associated lipocalin (NGAL), also known as lipocalin2, is a $25-\mathrm{kDa}$ protein belonging to the lipocalin superfamily. NGAL was originally found to be stored in specific granules of human neutrophils and includes a group of about 20 small secreted lipoproteins that can interact with specific ligands. These lipoproteins act as transporters of small hydrophobic substances, such as prostaglandins, retinoids, arachidonic acid, hormones, and fatty acids (5). In humans, elevated levels of NGAL expression have been detected in breast cancers (6), in adenocarcinomas of the lung, colon, liver, and pancreas $(7,8)$, and in ovarian cancers (9). NGAL is currently one of the most interesting and enigmatic proteins involved in tumor development (10). Previous studies have shown that NGAL has a role in extracellular matrix remodeling and can protect matrix metalloproteinase-9 (MMP-9) from degradation by interacting with NGAL $(11,12)$. In patients with breast cancer, a high-molecular-weight complex consisting of NGAL and MMP-9 has been found in urine samples (13), and elevated NGAL expression is associated with decreased survival (14). Elevated NGAL expression is also associated with increased tumor-cell motility $(15,16)$. Furthermore, NGAL has been shown to stabilize MMP-9, a critical enzyme required for extracellular matrix remodeling during tumor growth and metastasis $(11,17)$. NGAL may also promote breast cancer progression by regulating epithelial-to-mesenchymal transition (16). However, the biological roles of NGAL in cancer cells are incompletely understood.

In the present study, we examined NGAL expression in OSCC by immunohistochemical staining and Western blot analysis to gain further insight into the biologic roles of NGAL in OSCC. 
Table I. Characteristics of the patients with OSCC.

\begin{tabular}{lc}
\hline Total (n=30) & No. \\
\hline Mean age, years (range) & $63(39-83)$ \\
$\leq 63$ & 16 \\
$>63$ & 14 \\
Gender & \\
Male & 22 \\
Female & 8 \\
Primary & \\
T1/T2 & 20 \\
T3/T4 & 10 \\
Regional lymph nodes & \\
N0 & 17 \\
N1 & 6 \\
N2 & 7 \\
Stage & \\
I/II & \\
III/IV & 15 \\
Histopathology & 15 \\
Well differentiated & \\
Moderately differentiated & \\
Poorly differentiated & 5 \\
\hline
\end{tabular}

\section{Materials and methods}

Patients and surgical specimens. Surgical specimens were obtained from 40 patients who were treated in the Department of Oral and Maxillofacial Surgery, Hyogo College of Medicine. Five cases of normal mucosa (normal tissue), 5 cases of leukoplakia of the tongue, and 30 cases of tongue cancer (tumor tissue) were studied. Information on gender, age, stage of disease, and histopathological factors were retrieved from the patients' medical records. The patients' clinical characteristics are summarized in Table I. Tumor differentiation was evaluated according to the histological criteria of the guidelines of the World Health Organization Pathological Classification of Tumours (18).

Immunohistochemical and immunocytochemical staining. Tumor tissues were fixed in $10 \%$ formalin, embedded in paraffin, cut into $4-\mu \mathrm{m}$-thick sections, and stained with hematoxylin and eosin (H\&E) for histological examination. Immunohistochemical examinations were performed by the avidin-biotin-peroxidase complex (ABC) staining method. Briefly, the specimens were treated with $3 \% \mathrm{H}_{2} \mathrm{O}_{2}$ in methanol for $5 \mathrm{~min}$ to block endogenous peroxidase activity. The specimens were then washed and treated with $1 \%$ normal horse serum in PBS for $15 \mathrm{~min}$. After washing with PBS, the specimens were incubated with monoclonal antibodies against hNGAL (R\&D Systems Inc., Minneapolis, MN, USA). After washing again with PBS, the specimens were incubated with ABC complex solution (Vectastain, Vector Labs, Burlingame, CA, USA) at room temperature for $15 \mathrm{~min}$. The specimens were washed with PBS, applied biotinylated goat anti-mouse IgG (Vector), and incubated for $30 \mathrm{~min}$ at room temperature. Then, the specimens were treated with a substrate solution containing 3,3'-diaminobenzidine tetrahydrochloride (Wako Pure Chemical Industries, Ltd., Osaka, Japan) and $\mathrm{H}_{2} \mathrm{O}_{2}$ for about $5 \mathrm{~min}$. Finally, the specimens were counterstained with hematoxylin, dehydrated, and mounted with MountQuick (Daido Sangyo Co., Ltd). NGAL-positive samples were defined as those showing brown stain in the cytoplasm.

Immunohistochemical evaluation was performed by the method of Moniaux et al (19). All slides were observed under an Olympus light microscope, and representative photographs were taken. The intensity of immunoreactivity for NGAL was scored. The staining intensity was graded on a scale of 0 to $3+$ (0 for no staining; $1+$ for weak immunoreactivity; $2+$ for moderate immunoreactivity; and $3+$ for strong immunoreactivity). The percentage of cells with positive membranous and cytoplasmic staining for NGAL was graded as follows: grade 0 intensity (undetectable or negligible expression), no tumor cells positive for immunoreactivity; grade $1+$ intensity (low expression), $1-25 \%$ positive; grade $2+$ intensity (intermediate expression), 26-50\% positive; grade 3 intensity (high expression), $51-75 \%$ positive; and grade 4 intensity (high expression), $\geq 76 \%$ positive. The staining intensity score and the percent immunoreactivity score were multiplied to obtain a composite score. The value of the composite score ranged from a minimum of 0 to a maximum 12. The Mann-Whitney U-test was used to compare composite scores among the groups (normal oral mucosa, leukoplakia, and OSCC). Differences between the groups were defined as being statistically significant at P-value $<0.05$.

Cell culture and cell lines. Eight oral carcinoma cell lines (SCCKN, HSC-2, HSC-3, OSC-19, OSC-20, HOC-313, SCC-25, TSU) were grown in DMEM containing 10\% FBS and have been described previously (20-22). Cell lines were derived from OSCC with following grades of invasiveness: SCCKN, HSC-2, and OSC-20 cells from weakly invasive OSCC; HSC-3, OSC-19, and SCC-25 from mildly invasive OSCC; and HOC-313 and TSU from highly invasive OSCC. OSC-19, OSC-20, HOC-313, and TSU were kindly provided by Dr S. Kawashiri (Chief: E. Yamamoto, Department of Oral and Maxillofacial Surgery, Kanazawa University Graduate School of Medical Science, Kanazawa, Japan).

Protein preparation and Western blot analysis. The OSCC cells were lysed in a lysis buffer composed of PBS supplemented with $20 \mathrm{mM}$ Tris- $\mathrm{HCl}, \mathrm{pH} 8.0,1 \%$ NP40, $150 \mathrm{mM} \mathrm{NaCl}, 1 \mathrm{mM}$ EDTA, $10 \%$ glycerol, $0.1 \%$ $\beta$-mercaptoethanol, $0.5 \mathrm{mM}$ dithiothreitol, and a mixture of proteinase inhibitors consisting of $1 \mathrm{mM}$ phenylmethylsulfonyl fluoride, $10 \mu \mathrm{g} / \mathrm{ml}$ aprotinin, $5 \mu \mathrm{g} / \mathrm{ml}$ leupeptin, $5 \mathrm{mM}$ benzamidine, $1 \mu \mathrm{g} / \mathrm{ml}$ pepstatin, $2 \mu \mathrm{g} / \mathrm{ml}$ antipain hydrochloride (Boehringer Mannheim, Mannheim, Germany), $50 \mu \mathrm{M}$ 4-(2-aminoethyl)-benzenesulfonyl fluoride hydrochloride (Wako), $2 \mathrm{mM}$ sodium orthovanadate (Sigma-Aldrich Co., St. Louis, MO, USA), and $20 \mathrm{U} / \mathrm{ml}$ ulinastatin (Mochida Pharmaceutical, Tokyo, Japan). Lysate containing $30 \mu \mathrm{g}$ protein was electrophoresed on a $10-20 \%$ gradient SDS-PAGE mini gel (Dai-ichi Kagaku, Tokyo, 

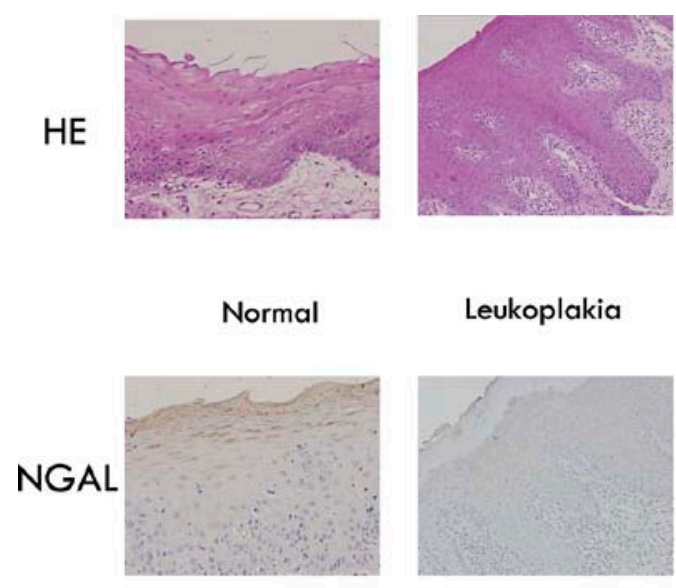
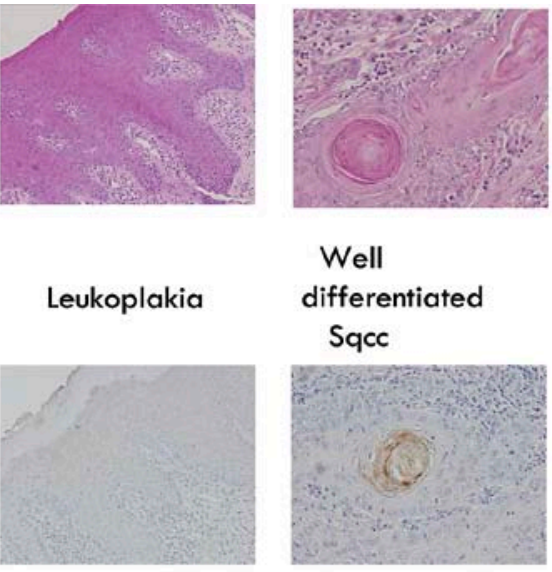
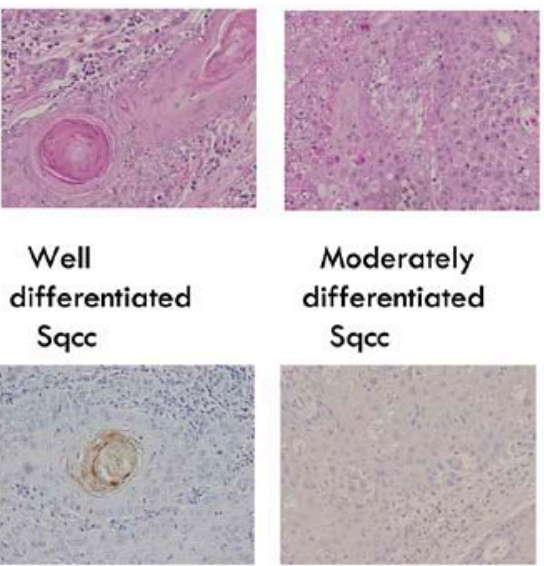

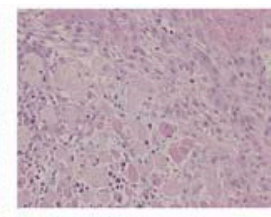

Poorly differentiated Sqce

\section{Correlation of NGAL expression with tongue cancer progression}

Figure 1. A representative immunohistochemical analysis of NGAL during the pathogenesis of OSCC. Representative sections of formalin-fixed, paraffinembedded tissues were immunolabeled with anti-NGAL monoclonal antibody, using the horseradish peroxidase detection method with DAB substrate. Brown product indicates positive immunoreactivity. NGAL expression in normal oral mucosa, leukoplakia, and well-, moderately, and poorly differentiated squamous cell carcinomas. The normal and leukoplakia tissue samples both show weak staining in the basal cell layer. In oral cancer, NGAL expression was strongest in well-differentiated tumors, weaker in moderately differentiated tumors, and absent in poorly differentiated tumors (top and bottom panels, original magnification, $\times 200$ ). All sections were counterstained with hematoxylin.

Japan) and blotted onto a PVDF membrane using Multiphor II (Amersham Pharmacia Biotech, Buchinghamshire, UK) for $30 \mathrm{~min}$. The blotted membrane was blocked with $5 \%$ skim milk in $10 \mathrm{mM}$ Tris- $\mathrm{HCl}, \mathrm{pH} \mathrm{7.2,} \mathrm{containing} 150 \mathrm{mM}$ $\mathrm{NaCl}$ and $0.5 \%$ Tween-20 and incubated with the primary antibodies $(0.1-1 \mu \mathrm{g} / \mathrm{ml})$ described below at $4^{\circ} \mathrm{C}$ for $16 \mathrm{~h}$. The membrane was then incubated with the alkaline phosphataseconjugated secondary antibodies $(0.02 \mu \mathrm{g} / \mathrm{ml})$ described below for $4 \mathrm{~h}$ at room temperature. The membrane was rinsed and treated with nitroblue tetrazolium (Sigma-Aldrich) and 5-bromo-4-chloro-3-indolyl phosphate (Sigma-Aldrich) to visualize protein bands. The primary antibodies used were mouse anti-hNGAL (R\&D Systems), mouse polyclonal antibodies against human actin (Santa Cruz Biotechnology, Santa Cruz, CA, USA), and monoclonal antibodies against EGFR, E-cadherin, and N-cadherin (all products, Santa Cruz). The secondary antibodies used were anti-mouse immunoglobulins conjugated with alkaline phos-phatase (Santa Cruz).

Immunofluorescence microscopy. The primary antibodies used in this study included goat anti-hNGAL (R\&D Systems) and mouse polyclonal antibodies against human actin (Santa Cruz). Cultured cells were washed PBS(-) 3 times and fixed in $3.7 \%$ paraformaldehyde for $15 \mathrm{~min}$ at room temperature. After blocking with $1.0 \%$ bovine serum albumin and the primary antibody at $4^{\circ} \mathrm{C}$ overnight, the cells were washed and incubated with anti-goat IgG-FITC or anti-mouse IgG-phalloidin (Invitrogen), followed by counterstaining with 4',6-diamidino2-phenylindole (DAPI). Finally, fluorescence images were acquired by using a confocal laser microscope, LSM 510 version 3.2 (Carl Zeiss Co., Ltd., Oberkochen, Germany).

Gelatin zymography. Gelatin zymography was performed as previously described by Narisawa-Saito et al (23). Cells $\left(1 \times 10^{8}\right)$ of each cell line were cultured for $24 \mathrm{~h}$ in growth medium and were then cultured for $24 \mathrm{~h}$ in serum-free medium. The conditioned medium was collected, concentrated 20 times, and separated through pre-cast gel with a Gelatin Zymography Kit (Primary Cell Co., Ltd., Sapporo, Japan). Gels were rinsed in washing buffer $(50 \mathrm{mM}$ Tris- $\mathrm{HCl}$, $\mathrm{pH} 7.5,2.5 \%$ Triton $\mathrm{X}-100)$ at room temperature for $1 \mathrm{~h}$ and incubated for $24 \mathrm{~h}$ at $37^{\circ} \mathrm{C}$ in incubation buffer $(50 \mathrm{mM}$ Tris- $\mathrm{HCl}, \mathrm{pH} 7.5,10 \mathrm{mM} \mathrm{CaCl}_{2}$, and $150 \mathrm{mM} \mathrm{NaCl}$ ). The gels were then fixed and stained with $0.25 \%$ Coomassie Blue R250. After destaining, gelatinolytic activity was visualized as a clear band against a blue background of stained gelatin. MMP-9 and MMP-2 were each detected as clear bands.

\section{Results}

Immunostaining for NGAL in normal epithelium, leukoplakia, and OSCC. To investigate the association between NGAL expression and the pathogenesis of oral cancer, NGAL expression patterns in oral cancers were investigated by immunohistochemistry. Forty formalin-fixed samples of normal tissue $(n=5)$, leukoplakia $(n=5)$, well-differentiated tongue cancer $(n=18)$, and moderate-poorly differentiated tongue cancer $(n=12)$ were studied immunohistochemically with the use of hNGAL monoclonal antibodies (Table I). In normal epithelium of oral mucosa, weakly positive staining for NGAL was located in limited areas of the epithelium. Well-differentiated cells in the superficial layers were weakly positive, but basal-layer cells were negative for NGAL. In leukoplakia, epithelial cells of the parabasal layers showed weakly positive staining for NGAL. In OSCC, strongly positive staining was observed in cancer cells. OSCC showed diverse staining patterns, ranging from strong homogeneous staining of most tumor cells to only focal staining of tumor cells (Fig. 1). NGAL expression was obviously associated with the degree of differentiation of OSCC. 


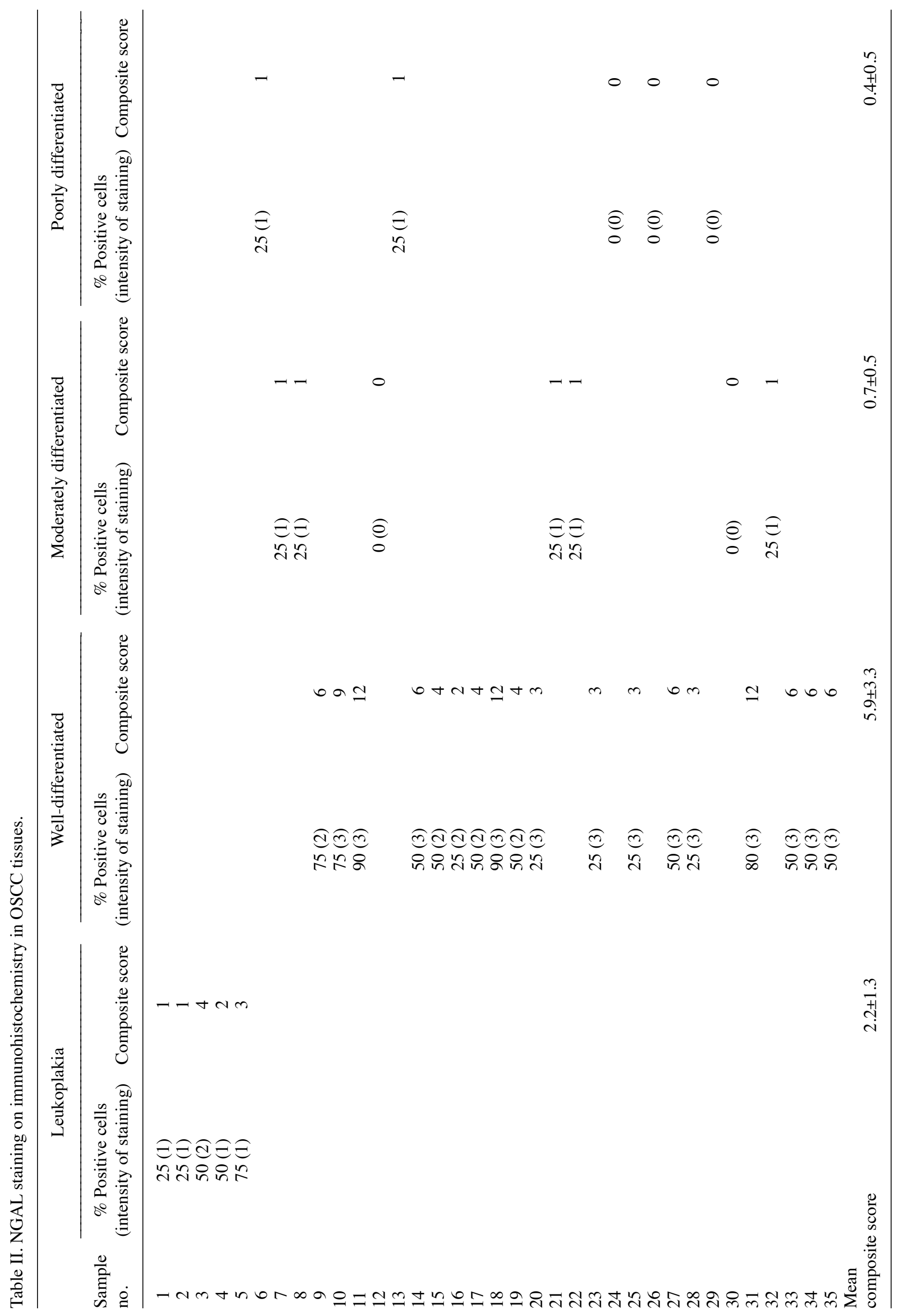




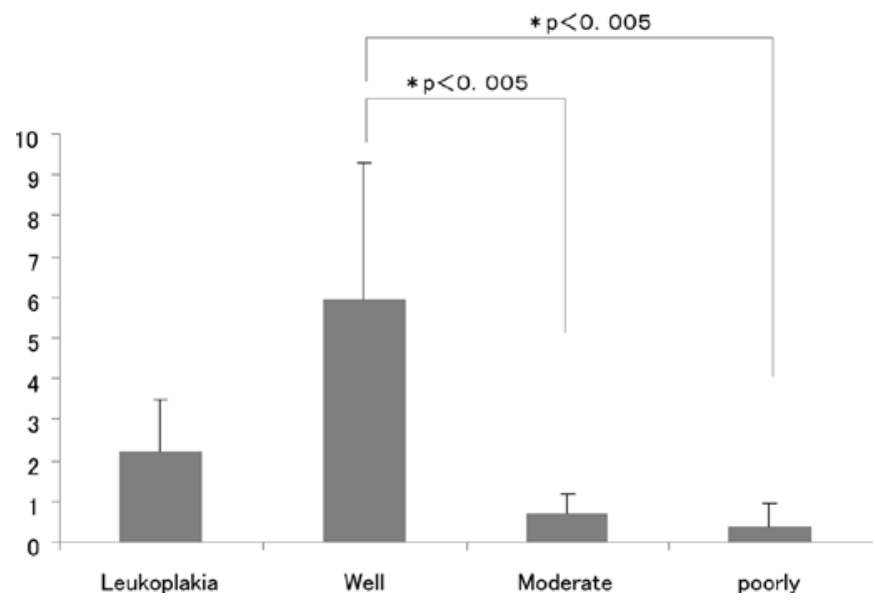

Figure 2. Mean composite score of NGAL levels in leukoplakia and well-, moderately, and poorly differentiated OSCCs. The mean composite score was highest in well-differentiated OSCC and differed significantly $(\mathrm{p}<0.005)$ between well-differentiated and moderately differentiated OSCCs as well as between well-differentiated and poorly differentiated OSCCs. Data are means \pm SD. ${ }^{*} \mathrm{p}<0.005$.

All well-differentiated OSCCs showed strongly positive staining. The staining intensity and number of positive cells were directly associated with the degree of differentiation of cells inside the cancer nests, with stronger staining in well-differentiated areas than in poorly differentiated areas. The mean composite score was 5.9 for well-differentiated OSCC, 0.7 for moderately differentiated OSCC, and 0.4 for poorly differentiated OSCC (Table II). The composite scores differed significantly $(\mathrm{p}<0.005)$ between well-differentiated and moderately differentiated OSCC, as well as between well-differentiated and poorly differentiated OSCC (Fig. 2). However, the parenchymal cells surrounding the cancer pearl were negative for NGAL. The moderately differentiated OSCCs showed very focal staining in a small subset of tumor cells. The poorly differentiated OSCCs were all negative for NGAL. These results showed that NGAL expression in welldifferentiated OSCC was directly associated with the degree of differentiation, with well-differentiated tumors showing the strongest expression and poorly differentiated tumors showing the weakest expression.

NGAL expression in OSCC cell lines. Since different degrees of NGAL expression were seen in tumor tissues, we examined the expression of NGAL in OSCC cell lines. Western blot revealed high expression levels of EGFR protein in all OSCC cell lines, consistent with the fact that nearly $90 \%$ of OSCC cell lines express EGFR (24). The pathological degree of differentiation of most cultured cell lines differs from that of primary cancer tissues and tumors transplanted to nude mice. We therefore analyzed in vitro E-cadherin expression and colony morphology patterns in addition to NGAL expression as indices of differentiation (Fig. 3). Tight-junctional colony morphology of SCCKN, HSC-2, and OSC-20, as well as mildly junctional colony morphology of HSC-3, OSC-19, and SCC-25 were associated with clear bands of E-cadherin, but fibroblastic morphology of HOC-313 and TSU was not associated with E-cadherin expression. In contrast, HOC-313 expressed $\mathrm{N}$-cadherin, confirming the loss of E-cadherin expression and suggesting the involvement of epithelial-
(A)

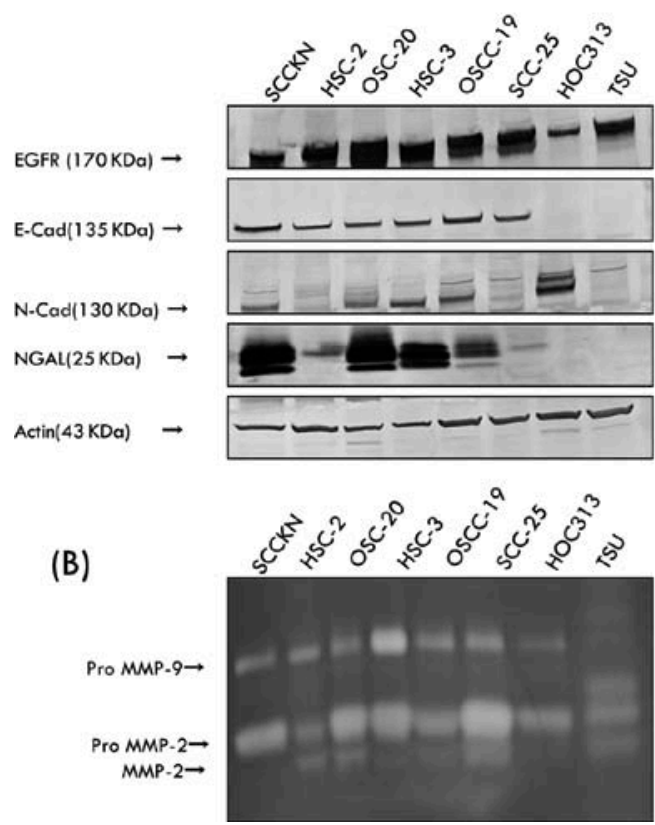

(C)
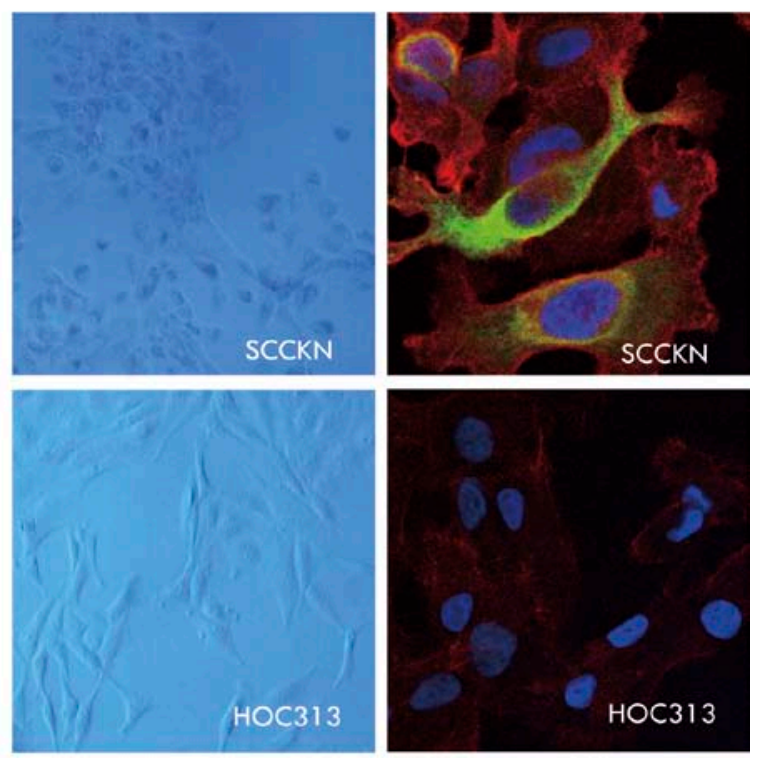

Figure 3. Western blot analysis of NGAL in a panel of oral cancer cell lines (A). Protein lysates from 8 oral cancer cell lines were resolved on a $15 \%$ SDSPAGE gel and transferred to a PVDF membrane. After blocking with 5\% BSA, the blot was hybridized with EGFR, E-cadherin, N-cadherin, anti-NGAL antibodies, and $\beta$-actin to demonstrate loading of proteins in all lanes. Gelatin zymographic analysis of matrix metalloproteinase activity in eight oral carcinoma cell lines (B). Immunocytochemical staining of SCCKN (well-differentiated) and HOC 313 (poorly differentiated) are shown (C). Blue, nuclear (DAPI); red, actin (phalloidin); green, NGAL (FITC). 
mesenchymal transition. HOC-313 cells are highly invasive and show spindle morphology without E-cadherin expression. Expression of NGAL was detected in all cell lines except for HOC-313 and TSU. HSC-2 and SCC-25 showed weakly positive NGAL expression. SCCKN and HOC-313 were stained immunocytochemically. Similar to the results of Western blot analysis, SCCKN, which showed weak invasiveness, was strongly positive for NGAL, whereas HOC-313 negatively stained for NGAL.

Finally, we examined the relation between NGAL and MMP-9 expression. MMP-9 expression was detected in all OSCC cell lines, and the intensities of MMP-9 expression were consistent with those of E-cadherin and NGAL expression.

\section{Discussion}

The TNM classification proposed by the Union Internationale Contre le Cancer (25) is a good system for describing the condition of cancer patients. However, this system can not predict the outcomes of individual patients or describe the biologic characteristics of tumor cells. It is therefore important to look for new objective prognostic factors that provide additional information about the biological characteristics of tumors. Invasion and metastasis are considered the most crucial characteristics of malignant tumors. Evaluation of the characteristics of cancer cells may facilitate selection of the best treatment strategy.

Matrix metalloproteinases are known to have important roles in extracellular matrix remodeling during tumor invasion. In vitro, the formation of $\mathrm{MMP} /$ neutrophil gelatinase-associated lipocalin (NGAL) complexes protects MMP-9 from autodegradation (11), and the human MCF-7 cancer cell line, which overexpresses NGAL, is associated with increased expression levels of MMP-9 (26). As for esophageal carcinoma, Li et al (27) demonstrated that down-regulation of NGAL expression in esophageal cells significantly suppresses MMP-9 activity and markedly increases the invasiveness of these cells in nude mice. Taken together with the results of our study, NGAL in conjunction with MMP-9 appears to play an important role in the invasiveness and progression of squamous cell carcinoma.

Deregulated expression of NGAL has been reported in several benign and malignant diseases. In our immunohistochemical study, NGAL showed diverse staining patterns in OSCC. The well-differentiated OSCCs in our series showed strongly positive staining for NGAL in the cancer pearl. In contrast, NGAL expression was negative in poorly differentiated OSCC and peripheral tumor cells of welldifferentiated OSCC. Similar results have been obtained in esophageal carcinoma (28). In normal epithelium, superficial well-differentiated cells were weakly positive for NGAL, but basal-layer cells were consistently negative for NGAL. In general, well-differentiated cells in superficial layers of normal epithelium have differentiated terminally from basal layer cells. Our results together with those of previous studies suggest that the up-regulation of NGAL induces not only carcinogenesis (i.e., the development of OSCC), but also the differentiation of OSCC. Lipocalins have well-documented roles in cell proliferation and differentiation (29). Mallbris et al (30) reported that NGAL was strongly induced in skin disorders associated with dysregulated epithelial differentiation. In OSCC, loss of terminal differentiation is one of the characteristics of cancer cells in well-differentiated tumors, and the manner in which cancer cells differentiate differs considerably from that of normal keratinized squamous epithelium (31). Since only weak NGAL expression was present in normal oral epithelium, up-regulation of NGAL in OSCC might imply dysregulated differentiation of cancer cells. Binding of lipocalins to specific receptors and subsequent receptor-mediated intracellular signaling might also have important roles in carcinogenesis.

In the tumor microenvironment, one of the earliest changes preceding the onset of a metastatic phenotype is an epithelial-mesenchymal transition associated with loss of E-cadherin expression, morphologic changes, and increased cellular motility (31). Thiery (32) recently demonstrated that NGAL can reverse this epithelial-mesenchymal transition, thereby diminishing the invasiveness of cancer cells. Our results show that in OSCC cell lines, NGAL is predominantly expressed in well-differentiated OSCCs, with virtually no expression in poorly differentiated OSCCs. These results suggest the hypothesis that loss of NGAL expression drives the progression of OSCC from well- to poorly differentiated tumors. Although further experiments are need to prove this hypothesis, if true, the development of recombinant NGALbased therapies might slow down epithelial to mesenchymal transformation and thereby inhibit the progression of OSCC.

In summary, NGAL was up-regulated in OSCC in a heterogeneous manner, and the expression level of NGAL correlated positively with the degree of cell differentiation. Moreover, the enzymatic activity of the NGAL/MMP-9 complex was much higher in OSCC than in normal oral mucosa and may thus have an important role in tumor invasion. Our findings suggest that NGAL is involved in the transformation and progression of OSCC.

\section{Acknowledgements}

Grant support: this study was supported by a Grant-in-Aid for Graduate Students, Hyogo College of Medicine, Grantsin-Aid for Scientific Research (B) (21390544) to M.U. and (C) (22592249) K.N. by the Japan Society for the Promotion of Science (JSPS), and a Grant-in-Aid for Young Scientists (B) (21890287) awarded to E.S. by The Ministry of Education, Culture, Sports, Science and Technology (MEXT). We would like to express our appreciation to Keiko Wakai and Takako Nanba for their expert assistance.

\section{References}

1. Hanahan D and Weinberg RA: The hallmark of Cancer. Cell 100: 57-70, 2000.

2. Pereira MC, Oliveira DT, Landman G and Kowalski LP: Histologic subtypes of oral squamous cell carcinoma: prognostic relevance. J Can Dent Assoc 73: 339-344, 2007.

3. Sudbø J, Bryne M, Kildal W, et al: Molecular based treatment of oral cancer. Oral Oncol 39: 749-758, 2003.

4. The GLOBOCAN 2002 database. http://www-dep.iarc.fr

5. Flower DR: The lipocalin protein family: structure and function. Biochem J 318: 1-14, 1996.

6. Stoesz SP, Friedl A, Haag JD, Lindstrom MJ, Clark GM and Gould MN: Heterogeneous expression of the lipocalin NGAL in primary breast cancers. Int J Cancer 79: 565-572, 1998. 
7. Friedl A, Stoesz SP, Buckley P and Gould MN: Neutrophil gelatinase-associated lipocalin in normal and neoplastic human tissue. Cell type-specific pattern of expression. Histochem J 31: 433-441, 1999.

8. Furutani M, Arii S, Mizumoto M, Kato M and Imamura M Identification of a neutrophil gelatinase-associated lipocalin mRNA in human pancreatic cancers using a modified signal sequence trap method. Cancer Lett 122: 209-214, 1998.

9. Bartsch $\mathrm{S}$ and Tschesche $\mathrm{H}$ : Cloning and expression of human neutrophil lipocalin cDNA derived from bone marrow and ovarian cancer cells. FEBS Lett 357: 255-259, 1995.

10. Bolignano D, Donato V, Coppolino G, et al: Neutrophil gelatinase-associated lipocalin (NGAL) in human neoplasias: a new protein enters the scene. Cancer Lett 288: 10-16, 2010.

11. Yan L, Borregaard N, Kjeldsen L and Moses MA: The molecular weight urinary matrix metalloproteinase (MMP) activity is a complex of galatinase B/MMP-9 activity by NGAL. J Biol Chem 276: 37258-37265, 2001

12. Sousa MM, do Amaral JB, Guimarāes A and Saraiva MJ: Up-regulation of the extracellular matrix remodeling genes, biglycan, neutrophil gelatinase-associated lipocalin, and matrix metalloproteinase-9 in familial amyloid polyneurophathy. FASEB J 19: 124-126, 2005.

13. Fernandez CA, Yan L, Louis G, Yang J, Kutok JL and Moses MA The matrix metalloproteinase-9/neutrophil gelatinase-associated lipocalin complex plays a role in breast tumor growth and is present in the urine of breast cancer patients. Clin Cancer Res 11: 5390-5395, 2005

14. Bauer M, Eickhoff JC, Gould MN, Mundhenke C, Maass N and Friedl A: Neutrophil gelatinase-associated lipocalin (NGAL) is a predictor of poor prognosis in human primary breast cancer. Breast Cancer Res Treat 108: 389-397, 2008.

15. Hu L, Hittelman W, Shmulevich I, et al: NGAL decreases E-cadherin-mediated cell-cell adhesion and increases cell motility and invasion through Rac1 in colon carcinoma cells Lab Invest 89: 531-548, 2009.

16. Yang J, Bielenberg DR, Kung AL, et al: Lipocalin 2 promotes breast cancer progression. Proc Natl Acad Sci USA 106: 3913-3918, 2009.

17. Kubben FJ, Sier CF, Zuidwijk K, et al: Clinical evidence for a protective role of lipocalin-2 against MMP-9 autodegradation and the impact for gastric cancer. Eur J Cancer 43: 1869-1876, 2007.

18. Barnes L, Eveson JW, Reichart P and Sidransky D: WHO Classification of Tumours. Pathology and Genetics Head and Neck Tumours. IARC Press, Lyon, 2005.

19. Moniaux N, Chakraborty S, Standop J, et al: Early diagnosis of pancreatic cancer: neutrophil gelatinase-associated lipocalin as a marker of pancreatic intraepithelial neoplasia. Br J Cancer 98: $1540-1547,2008$.
20. Urade M, Sugi M and Miyazaki T: Establishment of three bleomycin-resistant human carcinoma cell lines and their cross resistance to other antitumor agents. Cancer 61: 1501-1507, 1988

21. Kamata N, Chida K, Rikimaru K, Horikoshi M, Enomoto S and Kuroki T: Growth inhibitory effects of epidermal growth factor and overexpression of its receptors on human squamous cell carcinomas in culture. Cancer Res 46: 1648-1653, 1986.

22. Hoteiya T, Hayashi E, Satomura K, Kamata N and Nagayama N: Expression of E-cadherin in oral cancer cell lines and its relationship to invasiveness in SCID mice in vivo. J Oral Pathol Med 28: 107-111, 1999.

23. Narisawa-Saito M, Yoshimatsu Y, Kiyono T, et al: An in vitro multistep carcinogenesis model for human cervical cancer. Cancer Res 68: 5699-5705, 2008.

24. Sarkis SA, Abdullah BH, Abdul Majeed BA and Talabani NG: Immunohistochemical expression of epidermal growth factor receptor (EGFR) in oral squamous cell carcinoma in relation to proliferation, apoptosis, angiogenesis and lymphangiogenesis. Head Neck Oncol 2: 13, 2010.

25. Union Internationale Contre le Cancer. TNM Classification of malignant tumors. 5th edition. Wiley-Liss, New York, 1997.

26. Fernandez CA, Yang L, Louis G, et al: The matrix metalloproteinase-9/neutrophil gelatinase-associated lipocalin complex plays a role in breast tumor growth and is present in the urine of breast cancer patients. Clin Cancer Res 11: 5390-5395, 2005

27. Li EM, Xu LY, Cai WJ, Xiong HQ, Shen ZY and Zeng Y: Functions of neutrophil gelatinase-associated lipocalin in the esophageal carcinoma cell line SHEEC. Sheng Wu Hua Xue Yu Sheng Wu Wu Li Xue Bao (Shanghai) 35: 247-254, 2003.

28. Zhang HH, Xu LY, Wang ZY, et al: Upregulation of neutrophil gelatinase-associated lipocalin in oesophageal squamous cell carcinoma: significant correlation with cell differentiation and tumor invasion. J Clin Pathol 60: 555-561, 2007.

29. Flower DR: The lipocalin protein family: a role in cell regulation. FEBS Lett 354: 7-11, 1994.

30. Mallbris L, O'brien KP, Borregaard N, et al: Neutrophil gelatinase-associated lipocalin is a marker for dysregulated keratinocyte differentiation in human skin. Exp Dermatol 11: 584-591, 2002

31. van Dorst EB, van Muijen GN, Litvinov SV and Fleuren GJ: The limited difference between keratin patterns of squamous cell carcinoma and adenocarcinoma is explicable by both cell lineage and state of differentiation of tumor cells. J Clin Pathol 51: 679-684, 1998

32. Thiery JP: Epithelial-mesenchymal transitions in development and pathologies. Curr Opin Cell Biol 15: 740-746, 2003. 